\title{
Electrical Stabilization of Surface Resistivity in Epitaxial Graphene Systems by Amorphous Boron Nitride Encapsulation
}

Albert F. Rigosi, ${ }^{* \dagger \odot}$ Chieh-I Liu, ${ }^{\dagger, \S}$ Nicholas R. Glavin, ${ }^{\dagger}$ Yanfei Yang, ${ }^{\dagger, \|}$ Heather M. Hill, ${ }^{\dagger}$ Jiuning Hu, Angela R. Hight Walker, ${ }^{\dagger}$ Curt A. Richter, ${ }^{\dagger}$ Randolph E. Elmquist, ${ }^{\dagger}$ and David B. Newell

${ }^{\dagger}$ Physical Measurement Laboratory (PML), National Institute of Standards and Technology (NIST), 100 Bureau Drive, Gaithersburg, Maryland 20899, United States

${ }^{\ddagger}$ Materials and Manufacturing Directorate, Air Force Research Laboratory, 2941 Hobson Way, Wright-Patterson AFB, Dayton Ohio 45433, United States

${ }^{\S}$ Graduate Institute of Applied Physics, National Taiwan University, No. 1, Sec. 4, Roosevelt Road, Taipei 10617, Taiwan

"Joint Quantum Institute, University of Maryland, Bldg 224, Stadium Drive, College Park, Maryland 20742, United States

Supporting Information

ABSTRACT: Homogeneous monolayer epitaxial graphene (EG) is an ideal candidate for the development of millimeter-sized devices with single-crystal domains. A clean fabrication process was used to produce EG-based devices, with n-type doping level of the order of $10^{12} \mathrm{~cm}^{-2}$. Generally, electrical properties of EG, such as longitudinal resistivity, remain unstable when devices are exposed to air due to adsorption of molecular dopants, whose presence shifts the carrier density close to the Dirac point $\left(<10^{10} \mathrm{~cm}^{-2}\right)$ or into the ptype regime. Here, we report experimental results on the use of amorphous boron nitride (a-BN) as an encapsulation layer, whereby EG can maintain its longitudinal resistivity and have its carrier density modulated. Furthermore, we exposed 12 devices to controlled temperatures of up to $85{ }^{\circ} \mathrm{C}$ and relative humidity of up to $85 \%$ and reported that an approximately $20 \mathrm{~nm}$ a-BN encapsulation thickness is sufficient to preserve their longitudinal resistivity to

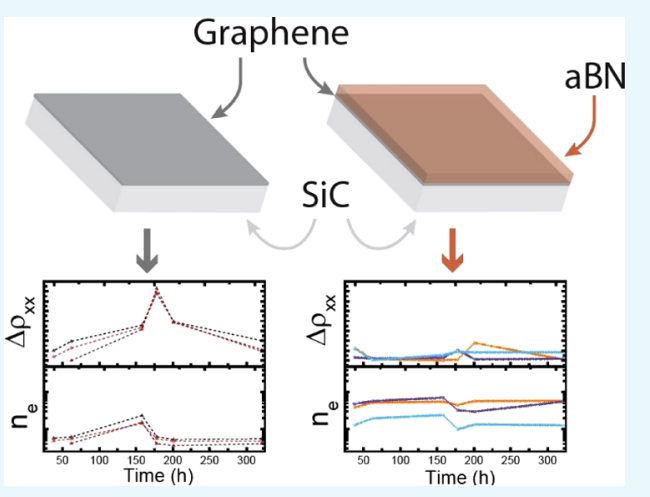
within $10 \%$ of the previously measured value. We monitored the electronic properties of our encapsulated and nonencapsulated EG samples by magnetotransport measurements, using a neodymium iron boron magnet. Our results have essential importance in the mass production of millimeter-scale graphene devices, with stable electrical properties.

\section{INTRODUCTION}

Graphene has been given significant attention over the recent years, in part for its desirable set of electrical properties. ${ }^{1-3}$ It has been shown that graphene grown on silicon carbide ( $\mathrm{SiC}$ ) displays properties most useful for the development of millimeter-sized, single-domain devices, particularly in the field of metrology. ${ }^{4-11}$ Experiments performed on millimeterscale devices indicate that epitaxially grown graphene (EG) can provide uniform transport properties over large areas, a prospect most promising when it comes to development of much larger graphene-based devices. ${ }^{12}$ Although properties of EG such as the longitudinal resistivity, carrier density, and mobility are generally good figures of merit to determine epitaxial graphene quality, ${ }^{13}$ these properties are desired to be controllable if such devices are to be mass produced.

When stored in air, the longitudinal resistivity of unprotected EG changes unpredictably with time, ${ }^{14-17}$ so it becomes necessary, specifically for electronic applications, to find an appropriate encapsulating material to both preserve the longitudinal resistivity of EG devices and to enable possible carrier-density control of the devices. The various studies addressing some of the effects of encapsulation on epitaxial graphene only address the effects from polymers and oxides. $^{18-22}$ Millimeter-scale encapsulation with boron nitride has not been explored mainly because with commonly used techniques involving mechanically exfoliated hexagonal boron nitride flakes, flake sizes do not typically exceed a few hundred micrometers, which does not satisfy the requirement of millimeter-sized coverage. Contrary to mechanical exfoliation, the technique of encapsulation with amorphous boron nitride (a-BN) by pulsed laser deposition ${ }^{23-25}$ can be used for millimeter-sized areas of EG and is performed at temperatures around $200{ }^{\circ} \mathrm{C}$. Here, we will report on the effectiveness of a$\mathrm{BN}$ as an encapsulation material on $\mathrm{EG}$ on the basis of transport measurements.

$\mathrm{EG}$ is grown on square $\mathrm{SiC}$ chips diced from $76 \mathrm{~mm} 4 \mathrm{H}-$ $\mathrm{SiC}(0001)$ semi-insulating wafers $\left(\mathrm{CREE}^{a}\right)$ of miscut similar to that in previous studies. ${ }^{12}$ Samples are prepared with a

Received: March 22, 2017

Accepted: May 17, 2017

Published: May 25, 2017 


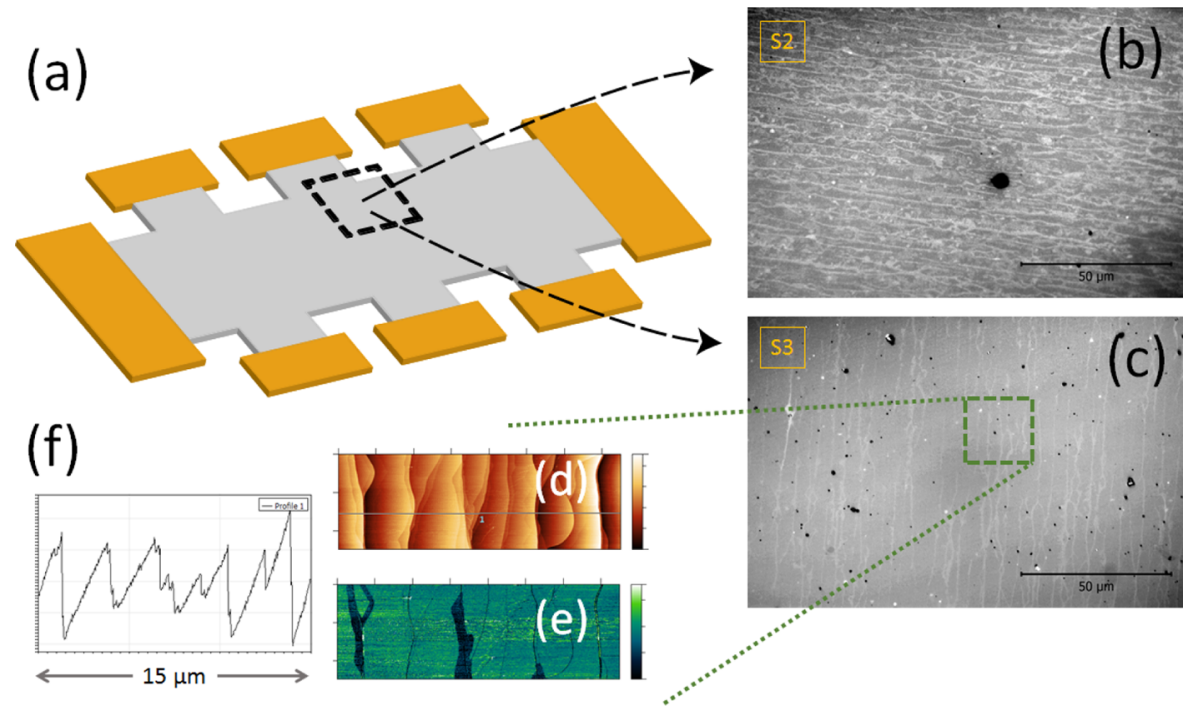

Figure 1. (a) An illustration of a Hall bar and the location at which optical and AFM images are taken. (b) Optical image, taken at 100 magnification, of an unprotected EG device (labeled S2D1) prior to the start of the experiment. (c) Optical image, taken at 100 $\times$ magnification, of an encapsulated EG device (labeled S3D2) prior to the start of the experiment. Within the dotted green region shown, (d) an AFM topographic image is acquired. (e) A phase image is also acquired to verify the coverage. (f) From the AFM topography, a line profile is shown to demonstrate the general sloped flatness of the terraces.

hydrofluoric acid rinse and cleaned with deionized water. They are then oriented such that the silicon face makes close contact to the polished pyrolytic graphite substrate (SPI Glas $22^{a}$ ), wherein the closeness is defined by the observation of Newton's rings. Epitaxial growth is then performed using a graphite-lined resistive-element furnace (Materials Research Furnaces Inc. ${ }^{a}$ ), with heating and cooling rates of approximately $1.5^{\circ} \mathrm{C} / \mathrm{s}$. The processing begins with a substrate cleaning at $1050{ }^{\circ} \mathrm{C}$ in a forming-gas environment ( $96 \% \mathrm{Ar}, 4 \% \mathrm{H}_{2}$ ) at $100 \mathrm{kPa}$ for 30 min. The forming-gas cleaning serves to hydrogenate the surface of the $\mathrm{SiC}$ chips. ${ }^{26,27}$ Processing continues with a chamber flush with Ar gas and filling with $100 \mathrm{kPa}$ Ar from a 99.999\% liquid Ar source before annealing at $1900{ }^{\circ} \mathrm{C}$.

Once EG is grown, the chips are placed in a home-built vacuum chamber, which is pumped down to at least $10^{-6} \mathrm{~Pa}$. Encapsulation of the EG with a-BN occurs via low-temperature pulsed laser deposition. The growth of the stoichiometric a-BN thin films has been extensively described in previous manuscripts $^{23,25}$ and allows for a thin film of insulating, high-quality boron nitride to be deposited on a multitude of substrates, including graphene. The process involves the use of a $\mathrm{KrF}$ laser to ablate a high-purity amorphous $\mathrm{BN}$ target in $6.66 \mathrm{~Pa}$ of ultrahigh-pressure nitrogen gas. The stoichiometric formation of the films is made possible by the gas phase reactions occurring between the ablated plasma and nitrogen background gas at that pressure, delivering the precursor gases to the substrate at precisely the right concentration and energies. ${ }^{28}$ The thickness of the $\mathrm{BN}$ film is controlled by the number of laser pulses striking the target, with a growth rate of approximately $1 \mathrm{~nm} \mathrm{BN}$ growth per 10 pulses. The substrate stage during growth was heated to $200{ }^{\circ} \mathrm{C}$, and upon completion of the a-BN deposition, the samples were allowed to cool to room temperature before removal of the EG/a-BN material stack. All encapsulations had thicknesses of approximately $20 \mathrm{~nm}$. Device fabrication follows a similar procedure as in the previous work. ${ }^{14} \mathrm{~A}$ detailed description of the fabrication procedure can be found in the Supporting Information.
All EG samples (and later, completed devices) were characterized by optical microscopy before and after deposition and after the experiment. Images were taken with a Nikon MM400 ${ }^{a}$ optical microscope and filtered to show high contrast at the $\mathrm{SiC}(0001)$ terrace boundaries, which form naturally during growth. On the basis of Figure 1c, the additional $20 \mathrm{~nm}$ of a-BN does not appear to significantly alter the quality of the images. Shortly, after graphene growth, atomic force microscopy (AFM) is used to determine the general coverage of EG. An Asylum Cypher ${ }^{a}$ was used to gather AFM images in tapping mode, one of which can be seen in Figure 1d. In addition to the topography of EG, phase images are also acquired. Topographic and phase images were acquired with a scan rate of $1 \mathrm{~Hz}$ and have a scan size of $15 \times 5 \mu \mathrm{m}^{2}$.

Raman spectra were recorded before deposition, after deposition, and at the end of the experiment, using a continuous-wave laser excitation at $514.5 \mathrm{~nm}$, with a commercial Renishaw InVia Raman ${ }^{a}$ microscope. The purpose of the Raman spectra was to verify that the deposition of $\mathrm{BN}$ did not either destroy or completely deteriorate the EG quality. Each spectrum for the five samples shown in Figure 2 is the average of a spectral map that was acquired to ensure reproducibility. The maps were collected at roughly the same location shown in Figure 1a, with $20 \mu \mathrm{m}$ steps, forming a 5 by 3 raster-style grid. There is no significant change in the $\mathrm{D}$ modes for the three chips encapsulated with $20 \mathrm{~nm}$ a-BN. Although the positions of the $2 \mathrm{D}\left(\mathrm{G}^{\prime}\right)$ mode mostly remained within a few wavenumbers $\left(2725 \mathrm{~cm}^{-1}\right)$ during the experiment, the deposition of a-BN appears to slightly shift the mode down by approximately $20 \mathrm{~cm}^{-1}$ for samples three and five, which indicates a slight increase in the electron density and an introduction of strain on the single crystal. ${ }^{29,30}$ The width of this mode for S5 is the only parameter that changes by more than a few wavenumbers $\left(\approx 30 \mathrm{~cm}^{-1}\right)$. Tables for values of the Raman positions and widths are provided in the Supporting Information. In the case of S4, the growth of graphene was much lower compared to that of S3 and S5. Therefore, one can expect that the susceptibility of S4 to Raman shifts is minimal 


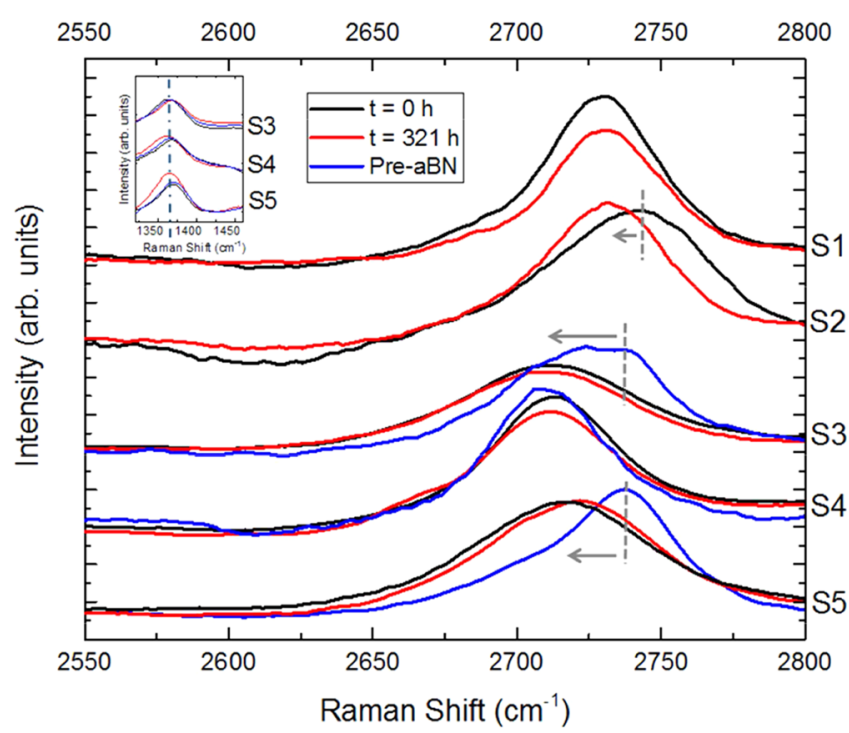

Figure 2. Raman spectra are shown for each of the five samples taken before (black curve) and after (red curve) the experiment, and in some cases, the Raman spectra are shown for the samples prior to a-BN deposition (blue curve). Curves are smoothed with an adjacent average of five points. The $\mathrm{SiC}$ background around the $2 \mathrm{D}\left(\mathrm{G}^{\prime}\right)$ mode is low, removing the need for subtraction. The inset shows the Raman spectral range around the $\mathrm{D}$ band of graphene. The inset curves have been subtracted with a background $\mathrm{SiC}$ Raman spectrum, taken with the same measuring parameters, to enhance clarity of the $\mathrm{D}$ mode seen at approximately $1365 \mathrm{~cm}^{-1}$. Each curve is the average of a Ramanmap acquisition consisting of 15 curves. The shifts for S2, S3, and S5 are shown as gray vertical lines.

due to the lower total surface area (more will be discussed on the impact of lower EG coverage).

Although data from the spectral range shown in the main part of Figure 2 had a low background, data from the spectral range of the $\mathrm{G}$ band is overwhelmed by the Raman response of the silicon carbide substrate. And on the other hand, the D band can be more easily extracted if the $\mathrm{SiC}$ background is subtracted, given that the background is not as strong in the spectral range neighboring the $\mathrm{D}$ band. When the $\mathrm{SiC}$ background is subtracted from each of the samples, one would expect either a flat spectrum or the $\mathrm{D}$ band at approximately $1350 \mathrm{~cm}^{-1}$. However, our Raman data show a broad peak, about $50 \mathrm{~cm}^{-1}$ in width, at the average peak position of $1364.6 \pm 3.3 \mathrm{~cm}^{-1}$. This data is shown as an inset to Figure 2. It is likely that this is a shifted D band ${ }^{29,31}$ because Raman data collected from an unprotected sample shows this peak, whereas data for a blank $\mathrm{SiC}$ chip deposited with a-BN lacks this peak.

\section{RESULTS AND DISCUSSION}

Once devices are fully fabricated and have their Raman spectra measured, they are prepared for magnetotransport measurements at room temperature. The setup is a four-point probe station, with an adjustable magnetic stage and a built-in microscope. With the addition of a Teflon block and neodymium iron boron (NIB) magnet, we can produce room temperature magnetic fields of up to $\pm 240 \pm 2 \mathrm{mT}$ (see Figure 3 for setup). Measurements and mapping of the $B$-field for this magnet were performed with a Lakeshore Gauss meter ${ }^{a}$ and are provided in the Supporting Information. The Hall voltage $\left(R_{x y}\right)$ is measured by aligning the device to the same marked spot on

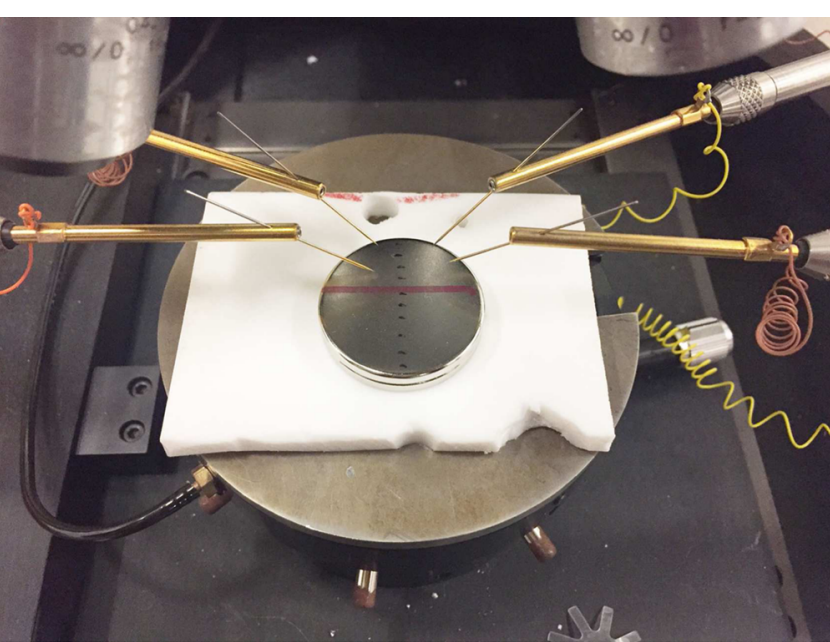

Figure 3. Photograph of the grade-N52 NIB magnet separated from the magnetic stage by a Teflon block in a four-point probe station. The NIB magnet is used to induce a strong-enough magnetic field for room-temperature measurements of the Hall voltage. The north and south maxima are measured to be $\pm 240 \pm 2 \mathrm{mT}$.

Table 1. Temperature and Relative Humidity Exposure Parameters.

$\begin{array}{lcc}\text { time }(\mathrm{h}) & \text { temperature }\left({ }^{\circ} \mathrm{C}\right) & \text { humidity }(\% \mathrm{RH}) \\ 0 & \text { ambient } & \text { ambient } \\ 0-21 & 85 & 0 \\ 21-38 & 22.2 & 85 \\ 38-62 & 22.2 & 45 \\ 62-158 & 22.2 & 45 \\ 158-178 & 85 & 85 \\ 178-201 & 85 & 0 \\ 201-321 & 22.2 & 45\end{array}$

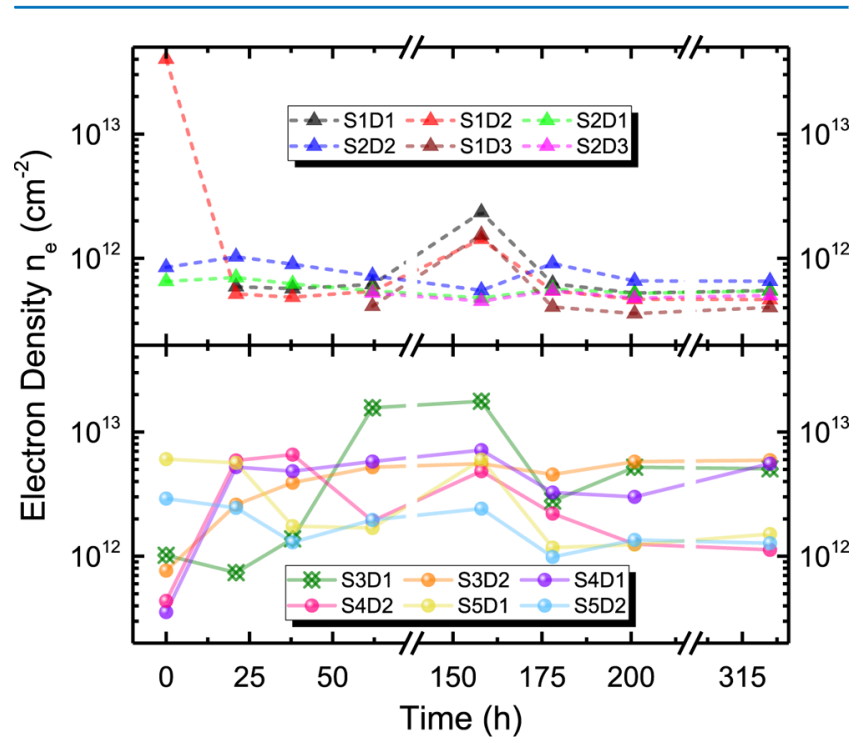

Figure 4. A semilogarithmic plot of the carrier density of 12 devices as a function of time is shown, with identical vertical scales. The top half of the graph shows the six unprotected EG devices as dashed curves, whereas the bottom half shows the six encapsulated devices as solid curves. Note that for the solid green curve (S3D1), the carrier density represents a hole density, as that device exhibited heavy p-doping after the fabrication process. The uncertainty of each point is smaller than the corresponding symbol used to indicate the value. 


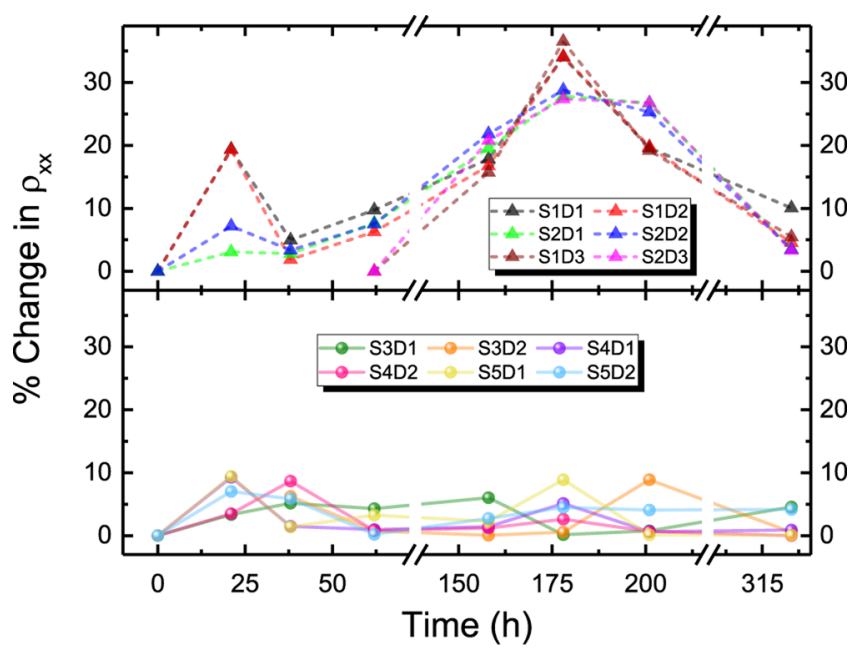

Figure 5. Graphs are shown for measurements of the percent change of longitudinal resistivity $\left(\rho_{x x}\right)$ as a function of time. The six unprotected and six encapsulated EG devices are represented in the top half (dashed curves) and bottom half (solid curves) of this graph, respectively. The greatly improved stability of $\rho_{x x}$ is demonstrated after each environmental exposure. The uncertainty of each point is smaller than the corresponding symbol used to indicate the value, and the vertical scales are identical to accentuate the improvements achieved with encapsulation.

the NIB magnet, and using Labview ${ }^{a}$ to run a script that collects $I-V$ curves from the probes and multimeter, with a maximum current of 1 and $0.05 \mu \mathrm{A}$ increments. By measuring $R_{x y}$ multiple times at each of the several magnetic-field points, one can extract the carrier density using

$$
n_{e}=\frac{1}{e\left(\frac{\mathrm{d} R_{x y}}{\mathrm{~d} B}\right)}
$$

Here, $e$ is the electron charge and the derivative is replaced by a slope, $\Delta R_{x y} / \Delta B$, taken from the best-fit line of all of the $R_{x y}$ voltages measured with a varying $B$-field. The longitudinal resistance, which we seek to stabilize and is marked $R_{x x}$ is measured in a similar electrical fashion, with probes being moved to new spots on the contacts. Each value is measured at least three times and averaged. Unlike $R_{x y}$, measurements at zero $B$-field are enough to determine the longitudinal resistivity, $\rho_{x x}$. One divides $R_{x x}$ by a length-to-width ratio that reflects the size and configuration of the Hall bar. In all cases for this experiment, the value of the length-to-width ratio is 2 . Additionally, to obtain the mobility of the device, one uses the following equation, which requires both the carrier density and longitudinal resistivity (note that the resistivity can be replaced by resistance, width $W$, and length $L$ of Hall bar)

$$
\mu=\frac{1}{e n_{e} R_{x x} \frac{W}{L}}
$$

These measurements are repeated for each of the 12 devices after various forms of environmental exposure. After a device is

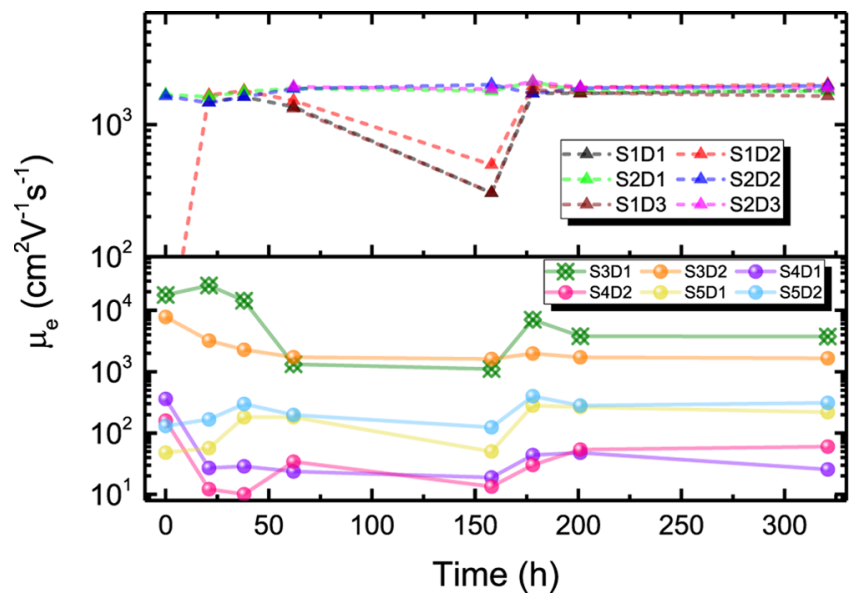

Figure 6. Carrier mobility for all 12 devices is represented here, with the long-dash dotted lines as the unprotected devices (top half) and the solid lines as the encapsulated devices (bottom half). The mobility at each point is calculated using the formula shown in the main text, using the data acquired for the electron density and longitudinal resistivity. The uncertainty of each point is smaller than the corresponding symbol used to indicate the value. The horizontal scale is kept fully intact for clarity.

first measured electrically, we expose it to a series of varied environmental conditions, which include changes in both temperature and relative humidity. We use a Thermotron ${ }^{a}$ testing chamber to expose samples. For temperatures and relative humidities, the chamber can maintain a particular parameter within $0.1 \%$ of the desired value, and so the used values are listed in Table 1.

When the carrier densities are measured, as shown in Figure 4 , a comparison is made between the two types of device, those that are unprotected ( $\mathrm{S} 1$ and $\mathrm{S} 2$ ) and those that are encapsulated with a-BN (S3, S4, and S5). Graphs with no breaks are provided in the Supporting Information. What becomes evident in the case of the unprotected EG is that although changes do occur with exposure to various environmental conditions, the changes are generally less than half of an order of magnitude. In the case of the a-BN-encapsulated devices, carrier densities can be tuned such that they reach and exceed an order of magnitude, depending on the device. However, in more ambient conditions, such as longer time frames of $62-158 \mathrm{~h}$ and $201-321 \mathrm{~h}$, the effects of environmental conditioning allow for repeated tuning of the carrier density. The changes in the carrier density can be attributed to the adsorption of water molecules on the edges of the device. Furthermore, water molecules can find their way into the EG/a-BN interface from the edges due to the hydrophilic buffer layer between EG and SiC. ${ }^{32}$ The ability of the a-BN encapsulation to assist in gateless modulation of the carrier density by use of extreme weathering is realized. Further studies with more varying parameters, such as thicker a-BN films, colder temperatures, and many additional devices are required to make a more definitive statement regarding the

\begin{tabular}{|c|c|c|c|c|c|c|c|c|c|c|c|}
\hline S1D1 & S1D2 & S1D3 & S2D1 & S2D2 & S2D3 & S3D1 & S3D2 & S4D1 & S4D2 & S5D1 & S5D2 \\
\hline 8889.8 & 9039.4 & 11484 & 5686.6 & 4504.5 & 6168.8 & 345.62 & 1054.8 & 49183 & 90147 & 21579 & 16523 \\
\hline 13.8 & 13.7 & 15.6 & 11.2 & 9.8 & 11.5 & 2.8 & 4.9 & 32.6 & 43.9 & 21.4 & 18.4 \\
\hline
\end{tabular}

Table 2. Initial Value of $\rho_{x x}(\Omega)$ and Associated Uncertainties. 
(a)
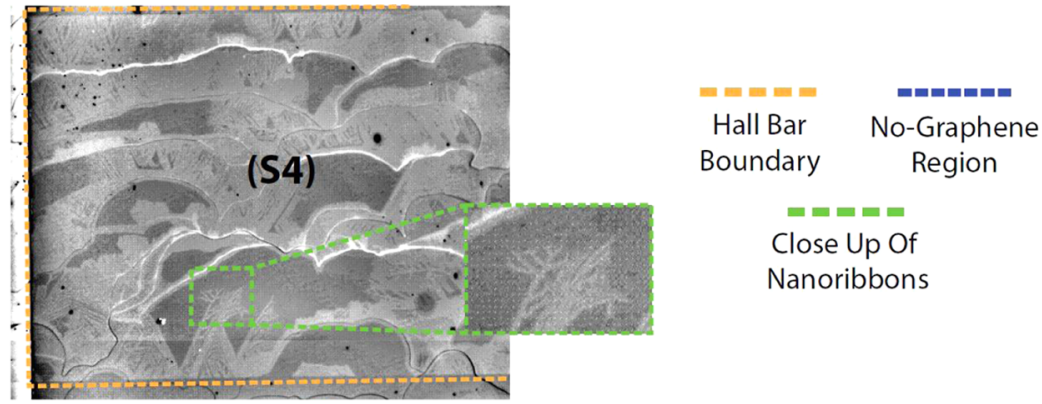

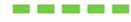

Close Up Of

Nanoribbons

(b)

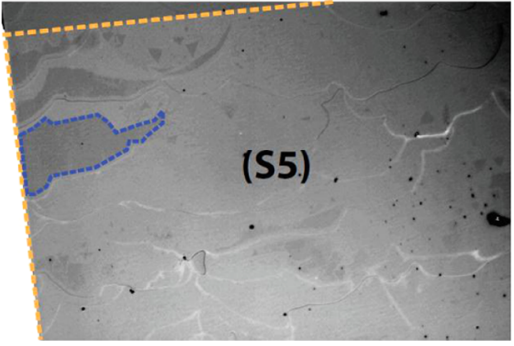

(c)

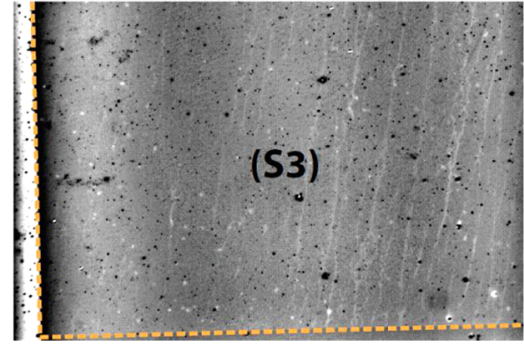

Figure 7. Sample regions are shown for an example device on each of the three EG chips which were deposited with a-BN. The three chips had different conditions of EG growth and the overall coverage varied from chip to chip. (a) The general coverage of EG on S4 was partially compared to that on S5 and S3. The inset is a zoom-in of a region of EG that, due to insufficient growth time, grew as nanoribbons that branch out and slowly converge into a uniform single crystal of graphene. (b) The same phenomenon is seen on S5 (as shown with a blue dotted line), but the growth shows significantly more coverage over the chip. (c) S3 shows no indication of the incomplete EG growth. On the contrary, the bright, vertical, edgelike features on the right half of the image are bilayers beginning to form along $\mathrm{SiC}$ terraces.

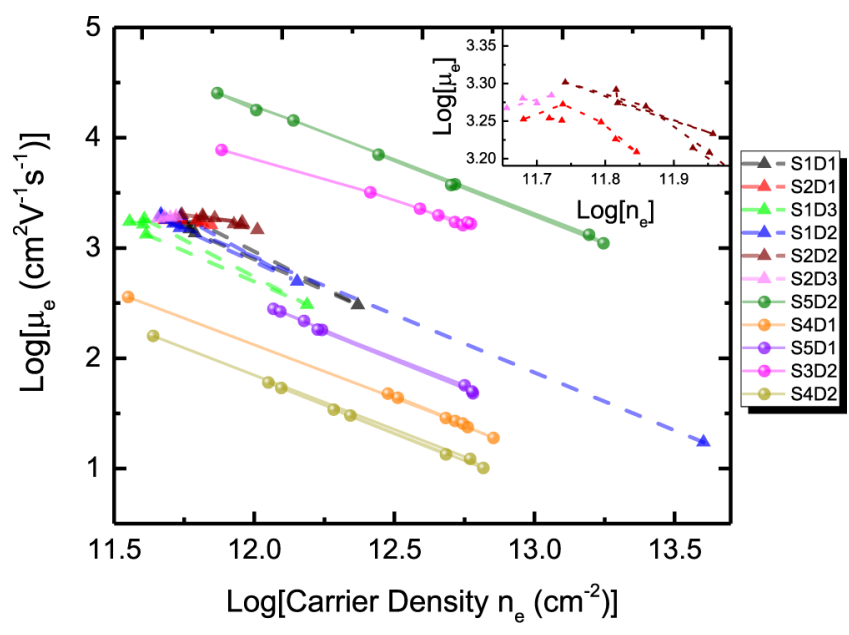

Figure 8. Graph plots the logarithm of mobility against logarithm of carrier density. Generally, the unprotected EG devices, represented by dashed lines and triangular data points, are confined to a smaller region of tunable mobility and carrier density, as opposed to the encapsulated devices, represented by dotted lines and circular data points. An inset is included to zoom in on the local parameter space behavior of three of the devices. S3D1 is excluded because of its pdoped behavior. The uncertainty of each point is smaller than the corresponding symbol used to indicate the value.

precision to which the carrier density can be modulated without a gate.

Measurements of the longitudinal resistivity show that the aBN encapsulation successfully preserves the value to within $10 \%$ of the previously measured value, regardless of the type of environmental exposure performed here. Figure 5 showing longitudinal or surface resistivity is graphed in a similar way, and Table 2 gives the initial values for the longitudinal resistivity, $\rho_{x x}$. This stable behavior is expected because the number of potential contaminants that could bind to the EG surface is no longer able to bind due to the presence of a-BN. It is worth noting that a pair of devices on a fourth chip were fabricated after the deposition of $10 \mathrm{~nm}$ of a-BN, but these devices failed to show stability and resembled the changes experienced by the unprotected devices. For more information, please see the Supporting Information.

In the top half of Figure 5, we observe a large change in the longitudinal resistivity, $\rho_{x x}$ in the unprotected EG devices. The values are shown as percentage changes from the previously measured values of $\rho_{x x}$. The sharpest drop in $\rho_{x x}$ occurs when devices are exposed to $85{ }^{\circ} \mathrm{C}$ and $85 \%$ relative humidity conditions for $23 \mathrm{~h}$. Two of the unprotected devices were introduced slightly later into the experiment (at $62 \mathrm{~h}$ ) to give us more statistics on general unprotected device behavior. The devices coated with a-BN, made from samples S3, S4, and S5, are in the bottom half of Figure 5. Unlike their unprotected counterparts, the encapsulated devices show a strong resistance to change when exposed to the same conditions, a feature that can be seen more clearly because the vertical scales of Figure 5 are identical for the top and bottom halves.

Five of the six devices did not deviate from this trend of sustained preservation of $\rho_{x x}$. The sixth, however, demonstrated a strange behavior at the start of the experiment. During 0-21 $\mathrm{h}$, with an exposure to $85{ }^{\circ} \mathrm{C}$ and $0 \%$ relative humidity, S3D2 developed bubbles on the surface, which seemed to be trapped by the thin film of a-BN (see Supporting Information for images and more details). With the formation of bubbles on the Hall bar, the corresponding drop of resistivity was from 1054.8 \pm 4.9 to $752.8 \pm 4.0 \Omega$. As the experiment progressed, continued formation did not occur, and the device remained within the $10 \%$ change-per-condition range. For this reason, the initial point was omitted and the curve renormalized but can be seen in its full form in the Supporting Information. The bubble formation decoupled a-BN from the EG surface and prevented any interfacial interactions, thus changing the resistivity for this initial measurement. Given that unprotected devices can change 
up to $35 \%$ of their previously held value, it is shown that approximately $20 \mathrm{~nm}$ of deposited a-BN is sufficient to encapsulate EG to preserve its longitudinal resistivity. Additionally, and to compare our results to another type of encapsulation, Parylene $\mathrm{C}$ is used to coat the two devices. In the case of the pinhole-free polymer, a film thickness greater than $500 \mathrm{~nm}$ was required to improve stabilization of the longitudinal resistivity. Another advantage a-BN has as an encapsulation material over other polymers, such as Parylene C, is that due to the thinner layer needed to achieve encapsulation, it could be applied to more devices in which top gates are implemented. More details on these measurements are presented in the Supporting Information.

By combining the data for $\rho_{x x}$ and carrier density, the mobility of each device can be monitored for each set of measurements performed. The resulting curves are shown in Figure 6 and follow similar trends to the carrier density. Although $\rho_{x x}$ does not change very much, as described earlier, we cannot expect the same from the mobility because it is mathematically dependent on the carrier density, which has been shown to be tunable with environmental conditioning. One also notices that the mobility is lower for some of the EG chips. Because of a poor miscut of the underlying $\mathrm{SiC}$ substrate, two of the encapsulated chips had a visibly lower yield of growth on the surface. Three encapsulated EG chips are shown in Figure 7, with optical images that were filtered to enhance the contrast between a single layer of EG and the substrate. Such stunted growth can easily lead to lower mobility and increased initial surface resistivity, as measured and shown in Table 2 of the main text. S4 has the highest longitudinal resistivity (by about an order of magnitude) compared to that of other samples.

In Figure 7, images are acquired, for example, on the devices on S3, S4, and S5 at the left drain contact, with the orange dotted line indicating the region just outside the boundary, wherein the EG edge and evidence of etched-away graphene is clearly noticeable. The matching contrast outside the Hall bar to the regions within the Hall bar show the SiC substrate. A closeup is provided in Figure 7a to show the stunted growth of the graphene on S4 and, by extension, on S5.

To show the extent of potentially controllable, gateless modulation, Figure 8 presents mobility as a function of carrier density for each device. Apart from a single data point, most curves for the unprotected EG devices remain relatively confined to the neighborhood of the inherent value of the carrier density and mobility. By having eventual control over the amount of carrier modulation, every device can be optimized to user-desired values regardless of the inherent value.

\section{CONCLUSIONS}

We performed experiments to determine whether the use of a$\mathrm{BN}$ as an encapsulation layer is sufficient to preserve the longitudinal resistivity of EG. Post deposition, we exposed six encapsulated devices and six unprotected devices to controlled temperatures of up to $85{ }^{\circ} \mathrm{C}$ and relative humidity of up to $85 \%$. Overall, a-BN encapsulation thickness of approximately $20 \mathrm{~nm}$ is sufficient to preserve the longitudinal resistivity within $10 \%$ of its last-measured, post-deposition value. Furthermore, data taken for carrier densities suggest a potential controllable method to tune carrier density by use of an environmental chamber. Our results have immediate applicability in the mass production of millimeter-scale graphene devices, with stable and optimized electrical properties for a wide range of engineering purposes.

\section{ASSOCIATED CONTENT}

\section{Supporting Information}

The Supporting Information is available free of charge on the ACS Publications website at DOI: 10.1021/acsomega.7b00341.

Epitaxial graphene growth preparation and fabrication, Raman data tables, NIB magnetic-field data, bubble formation for a specific device, main text graphs without axis breaks, graphene growth of sample S4, and a comparison to polymer encapsulation (PDF)

\section{AUTHOR INFORMATION}

\section{Corresponding Author}

*E-mail: albert.rigosi@nist.gov. Tel: +1 3019756572.

ORCID

Albert F. Rigosi: 0000-0002-8189-3829

Angela R. Hight Walker: 0000-0003-1385-0672

\section{Author Contributions}

A.F.R. designed the experiment. A.F.R., C.-I.L., N.R.G., Y.Y., H.M.H., and J.H. performed the experiments. Y.Y. and R.E.E. produced graphene samples. N.R.G. performed BN deposition. The manuscript was written through contributions of all authors. All authors have given approval to the final version of the manuscript.

\section{Funding}

Work done by Y.Y. was supported by federal grant \#70NANB12H185.

\section{Notes}

The authors declare no competing financial interest.

\section{ACKNOWLEDGMENTS}

A.F.R. and H.M.H. would like to thank the National Research Council's Research Associateship Program for the opportunity. We would like to thank Vishal Panchal for fruitful discussions. The work of C.-I.L. at NIST was made possible by arrangement with Prof. C.-T. Liang of National Taiwan University. The authors would like to thank Art Safriet of the University of Dayton Research Institute for the design of components for the a-BN growth chamber.

\section{ADDITIONAL NOTE}

${ }^{a}$ Commercial equipment, instruments, and materials are identified in this article to specify the experimental procedure adequately. Such identification is not intended to imply recommendation or endorsement by the National Institute of Standards and Technology or the U.S. government, nor is it intended to imply that the materials or equipment identified are necessarily the best available for the purpose.

\section{REFERENCES}

(1) Geim, A. K.; Novoselov, K. S. The rise of graphene. Nat. Mater. 2007, 6, 183-191.

(2) Novoselov, K. S.; Geim, A. K.; Morozov, S. V.; Jiang, D.; Zhang, Y.; Dubonos, S. V.; et al. Electric field effect in atomically thin carbon films. Science 2004, 306, 666-669.

(3) Novoselov, K. S.; Fal'ko, V. I.; Colombo, L.; Gellert, P. R.; Schwab, M. G.; Kim, K. A roadmap for graphene. Nature 2012, 490, 192-200.

(4) Fukuyama, Y.; Elmquist, R. E.; Huang, L.-I.; Yang, Y.; Liu, F.-H.; Kaneko, N.-H. Controlling the Fermi Level in a Single-Layer 
Graphene QHE Device for Resistance Standard. IEEE Trans. Instrum. Meas. 2015, 64, 1451-1454.

(5) Janssen, T. J. B. M.; Tzalenchuk, A.; Yakimova, R.; Kubatkin, S.; Lara-Avila, S.; Kopylov, S.; et al. Anomalously strong pinning of the filling factor $\mathrm{nu}=2$ in epitaxial graphene. Phys. Rev. B 2011, 83, No. 233402.

(6) Ribeiro-Palau, R.; Lafont, F.; Brun-Picard, J.; Kazazis, D.; Michon, A.; Cheynis, F.; et al. Quantum Hall resistance standard in graphene devices under relaxed experimental conditions. Nat. Nanotechnol. 2015, 10, 965-971.

(7) Tzalenchuk, A.; Lara-Avila, S.; Kalaboukhov, A.; Paolillo, S.; Syväjärvi, M.; Yakimova, R; et al. Towards a quantum resistance standard based on epitaxial graphene. Nat. Nanotechnol. 2010, 5, 186189.

(8) Real, M. A.; Lass, E. A.; Liu, F.-H.; Shen, T.; Jones, G. R.; Soons, J. A.; et al. Graphene epitaxial growth on $\mathrm{SiC}(0001)$ for resistance standards. IEEE Trans. Instrum. Meas. 2013, 62, 1454-1460.

(9) Lafont, F.; Ribeiro-Palau, R.; Kazazis, D.; Michon, A.; Couturaud, O.; Consejo, C.; et al. Quantum Hall resistance standards from graphene grown by chemical vapour deposition on silicon carbide. Nat. Commun. 2015, 6, No. 6806.

(10) Janssen, T. J. B. M.; Rozhko, S.; Antonov, I.; Tzalenchuk, A.; Williams, J. M.; Melhem, Z.; et al. Operation of graphene quantum Hall resistance standard in a cryogen-free table-top system. 2D Mater. 2015, 2, No. 035015.

(11) Jeckelmann, B.; Jeanneret, B. The quantum Hall effect as an electrical resistance standard. Rep. Prog. Phys. 2001, 64, 1603-1655.

(12) Yang, Y.; Cheng, G.; Mende, P.; Calizo, I. G.; Feenstra, R. M.; Chuang, C.; et al. Epitaxial Graphene Homogeneity and Quantum Hall Effect in Millimeter-Scale Devices. Carbon 2017, 115, 229-236.

(13) Novikov, S.; Lebedeva, N.; Pierz, K.; Satrapinski, A. Fabrication and Study of Large-Area QHE Devices Based on Epitaxial Graphene. IEEE Trans. Instrum. Meas. 2015, 64, 1533-1538.

(14) Yang, Y.; Huang, L.-I.; Fukuyama, Y.; Liu, F.-H.; Real, M. A.; Barbara, P.; et al. Low Carrier Density Epitaxial Graphene Devices on SiC. Small 2015, 11, 90-95.

(15) Wehling, T. O.; Novoselov, K. S.; Morozov, S. V.; Vdovin, E. E.; Katsnelson, M. I.; Geim, A. K.; et al. Molecular doping of graphene. Nano Lett. 2008, 8, 173-177.

(16) Ni, Z. H.; Wang, H. M.; Luo, Z. Q.; Wang, Y. Y.; Yu, T.; Wu, Y. H.; et al. The effect of vacuum annealing on graphene. J. Raman Spectrosc. 2010, 41, 479-483.

(17) Nomani, M. W. K.; Shields, V.; Tompa, G.; Sbrockey, N.; Spencer, M. G.; Webb, R. A.; et al. Correlated conductivity and work function changes in epitaxial graphene. Appl. Phys. Lett. 2012, 100, No. 092113.

(18) Lara-Avila, S.; Moth-Poulsen, K.; Yakimova, R.; Bjørnholm, T.; Fal'ko, V.; Tzalenchuk, A.; et al. Non-volatile photochemical gating of an epitaxial graphene/polymer heterostructure. Adv. Mater. 2011, 23, $878-882$.

(19) Robinson, J. A.; LaBella, M.; Trumbull, K. A.; Weng, X. J.; Cavelero, R.; Daniels, T.; et al. Epitaxial graphene materials integration: effects of dielectric overlayers on structural and electronic properties. ACS Nano 2010, 4, 2667-2672.

(20) Garces, N. Y.; Wheeler, V. D.; Hite, J. K.; Jernigan, G. G.; Tedesco, J. L.; Nepal, N.; et al. Epitaxial graphene surface preparation for atomic layer deposition of $\mathrm{Al}(2) \mathrm{O}(3)$. J. Appl. Phys. 2011, 109, No. 124304.

(21) Parmentier, F. D.; Cazimajou, T.; Sekine, Y.; Hibino, H.; Irie, H.; Glattli, D. C.; Kumada, N.; Roulleau, P. Quantum Hall effect in epitaxial graphene with permanent magnets. Sci. Rep. 2016, 6, No. 38393.

(22) Alaboson, J. M. P.; Wang, Q. H.; Emery, J. D.; Lipson, A. L.; Bedzyk, M. J.; Elam, J. W.; et al. Seeding atomic layer deposition of high-k dielectrics on epitaxial graphene with organic self-assembled monolayers. ACS Nano 2011, 5, 5223-5232.

(23) Glavin, N. R.; Muratore, C.; Jespersen, M. L.; Hu, J.; Hagerty, P. T.; Hilton, A. M.; et al. Amorphous Boron Nitride: A Universal,
Ultrathin Dielectric for 2D Nanoelectronics. Adv. Funct. Mater. 2016, 26, 2640-2647.

(24) Bresnehan, M. S.; Hollander, M. J.; Wetherington, M.; Labella, M.; Trumbull, K. A.; Cavalero, R.; et al. Integration of hexagonal boron nitride with quasi-freestanding epitaxial graphene: toward wafer-scale, high-performance devices. ACS Nano 2012, 6, 5234-5241.

(25) Glavin, N. R.; Jespersen, M. L.; Check, M. H.; Hu, J.; Hilton, A. M.; Fisher, T. S.; et al. Synthesis of few-layer, large area hexagonalboron nitride by pulsed laser deposition. Thin Solid Films 2014, 572, 245-250.

(26) Schardt, J.; Bram, C.; Müller, S.; Starke, U.; Heinz, K.; Müller, K. LEED structure determination of hexagonal $\alpha$-SiC surfaces. Surf. Sci. 1995, 337, 232-242.

(27) Seyller, T. Passivation of hexagonal SiC surfaces by hydrogen termination. J. Phys.: Condens. Matter 2004, 16, S1755-S1782.

(28) Glavin, N. R.; Muratore, C.; Jespersen, M. L.; Hu, J.; Fisher, T. S.; Voevodin, A. A. Temporally and spatially resolved plasma spectroscopy in pulsed laser deposition of ultra-thin boron nitride films. J. Appl. Phys. 2015, 117, No. 165305.

(29) Robinson, J. A.; Puls, C. P.; Staley, N. E.; Stitt, J. P.; Fanton, M. A.; Emtsev, K. V.; et al. Raman topography and strain uniformity of large-area epitaxial graphene. Nano Lett. 2009, 9, 964-968.

(30) Bissett, M. A.; Tsuji, M.; Ago, H. Strain engineering the properties of graphene and other two-dimensional crystals. Phys. Chem. Chem. Phys. 2014, 16, 11124.

(31) Ni, Z. H.; Chen, W.; Fan, X. F.; Kuo, J. L.; Yu, T.; Wee, A. T. S.; et al. Raman spectroscopy of epitaxial graphene on a $\mathrm{SiC}$ substrate. Phys. Rev. B 2008, 77, No. 115416.

(32) Zhou, H.; Ganesh, P.; Presser, V.; Wander, M. C. F.; Fenter, P.; Kent, P. R. C.; Jiang, D.; Chialvo, A. A.; McDonough, J.; Shuford, K. L.; Gogotsi, Y. Understanding controls on interfacial wetting at epitaxial graphene: experiment and theory. Phys. Rev. B 2012, 85, No. 035406. 